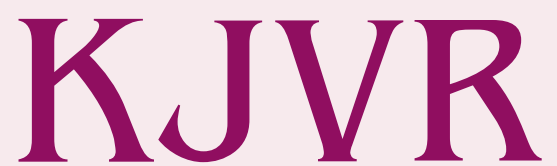

Korean Journal of Veterinary Research

\section{Case Report}

pISSN 2466-1384 · elSSN 2466-1392

Korean J Vet Res 2021;61(3):e27

https://doi.org/10.14405/kjvr.2021.61.e27

${ }^{*}$ Corresponding author:

Ji-Youl Jung

College of Veterinary Medicine and

Veterinary Medical Research Institute, Jeju

National University, Jeju 63243, Korea

Tel: +82-64-754-3364

Fax: +82-64-702-9920

E-mail: jungjy1982@jejunu.ac.kr

ORCID:

https://orcid.org/0000-0002-5938-2029

Conflict of interest:

The authors declare no conflict of interest.

Received: July 21, 2021

Revised: August 13, 2021

Accepted: August 17, 2021

\title{
Mesenchymal chondrosarcoma in the maxillary gingiva of a Maltese dog: a case report
}

\author{
So-Jeong Yim', Jinyong Lee ${ }^{2}$, Jae-Hoon Kim', Ji-Youl Jung ${ }^{1, *}$ \\ ${ }^{1}$ Veterinary Medical Research Institute, College of Veterinary Medicine, Jeju National University, \\ Jeju 63243, Korea \\ ${ }^{2}$ Malee Animal Hospital, Jeju 63067, Korea
}

A 13-year-old castrated Maltese dog was presented to a local animal hospital with an oral hemorrhage. An intraoral examination revealed an irregular proliferated lobular mass at the right side of the maxillary gingiva and hard palate. A surgically excised mass was requested for a histopathology examination. Histopathologically, the neoplastic foci were composed of biphasic morphologic patterns, such as primitive mesenchymal tissue and mature or immature cartilage tissue. Immunohistochemically, most of the neoplastic cells forming cartilaginous islands tested positive for S-100; the surrounding mesenchymal cells tested positive for vimentin. This paper describes a rare case of mesenchymal chondrosarcoma in the maxillary gingiva of a Maltese dog.

Keywords: Maltese dog; maxilla; gingiva; mesenchymal chondrosarcoma; case report

Chondrosarcoma is a malignant neoplasm in which tumor cells produce various amounts of neoplastic chondroid and fibrillary matrix, but not osteoid matrix [1]. This is the second most common primary tumor of bone in humans and dogs and accounts for approximately $5 \%$ to $10 \%$ of all canine primary bone tumors [2].

In dogs, chondrosarcoma occurs most often in medium to large breeds, particularly boxers, German shepherds, and golden retrievers, but is rare in small and giant breeds $[1,3]$. Moreover, the tumor is most common in middle-aged to older dogs; the mean age of the affected animals ranges from 5.9 to 8.7 years [3].

Mesenchymal chondrosarcoma is a rare subtype of chondrosarcoma among the World Health Organization classifications and accounts for approximately $1 \%$ of all chondrosarcomas in humans [4]. Histologically, mesenchymal chondrosarcomas are characterized by a transition from undifferentiated mesenchymal cells and an array of variously differentiated chondroid components [1]. On the other hand, chondrosarcoma is relatively rare in most domestic species. This paper presents a rare case of mesenchymal chondrosarcoma in the maxillary gingiva of a small-breed Maltese dog.

A 13-year-old castrated male Maltese dog was presented to a local animal hospital with an oral hemorrhage and swelling on the right side of his face. An intraoral examination revealed an irregular proliferated lobular mass at the entire right side of the gingival region and hard palate (Fig. 1). A radiography examination showed a soft tissue mass lesion in the right maxillary region without metastases to other organs. Blood analysis revealed mild anemia (hematocrit, 29.8\%; reference range, 35\%-56\%) and mild leukocytosis (21.8 L/ $\mu \mathrm{L}$; reference range, 5.0-20.0 L/ $\mu \mathrm{L})$. The serum biochemical results revealed moderate elevations in the alkaline phosphatase (113 U/L; reference range, $17-78 \mathrm{U} / \mathrm{L})$.

The dog did not respond after a 1-week treatment with steroids, and the edema- 


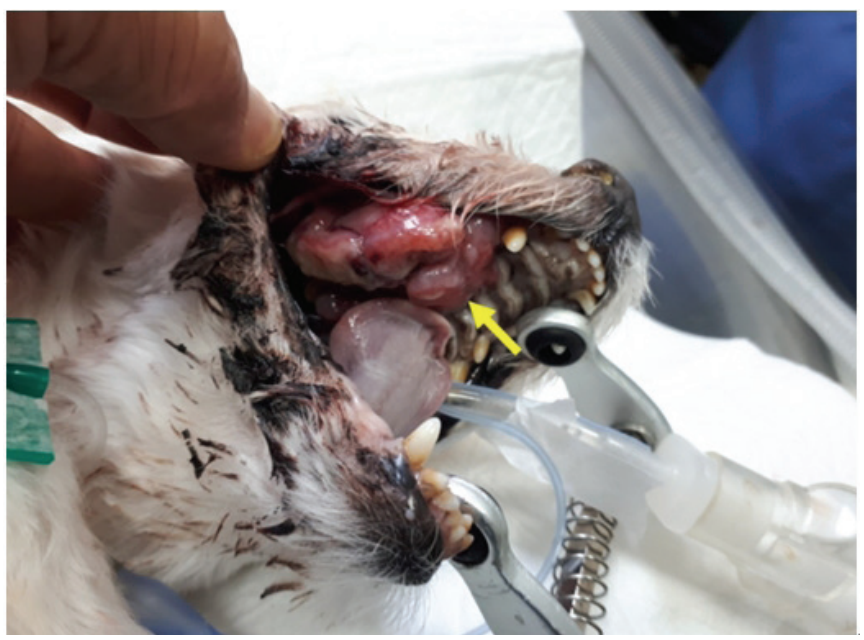

Fig. 1. Gross findings. Irregular proliferated lobular mass was noted at the entire right side of the maxillary gingiva and hard palate (arrow).

tous lesion worsened. The edema resulted in the caudal and dorsal displacement of the right eyeball. An incisional biopsy sample was presented to the Pathology Department of the Veterinary Medicine, Jeju National University, for a histopathological examination. The requested mass was approximately $3.5 \times$ $1.8 \times 1.8 \mathrm{~cm}$ in size. The cut surface was firm and tan to white.

The mass was fixed in 10\% neutral-buffered formalin, trimmed and processed according to standard protocols, and embedded in paraffin wax. The paraffin sections $(4 \mu \mathrm{m})$ were stained with hematoxylin and eosin and Alcian blue $\mathrm{pH} 1.0$ and 2.5) for the microscopic examination. To determine the origin of the tumor cells, replicated paraffin sections of the mass were also subjected to immunohistochemical analysis and incubated with the following primary antibodies: anti-vimentin (mouse monoclonal antibody, 1:100; Dako, Denmark) and anti-S-100 (rabbit polyclonal antibody, 1:400; Dako).

The microscopy examination showed that the neoplastic foci comprised a biphasic morphological pattern, such as primitive mesenchymal tissue and mature or immature cartilage tissues (Fig. 2A). The primitive mesenchyme was composed of round and oval or mild elongated immature neoplastic cells with high cellularity. These cells had scant pale eosinophilic cytoplasm and indistinct cellular borders with some mitotic figures. The cartilage areas were scattered throughout the lesions. The tumor cells were arranged irregularly within the lacunae of a pale, basophilic or eosinophilic, intercellular matrix (Fig. 2B). These neoplastic cells had abundant pale-staining cytoplasm, moderate anisokaryosis, and round vesicular nuclei with prominent nucleoli. Mitotic figures were rare. A small number of multinu-

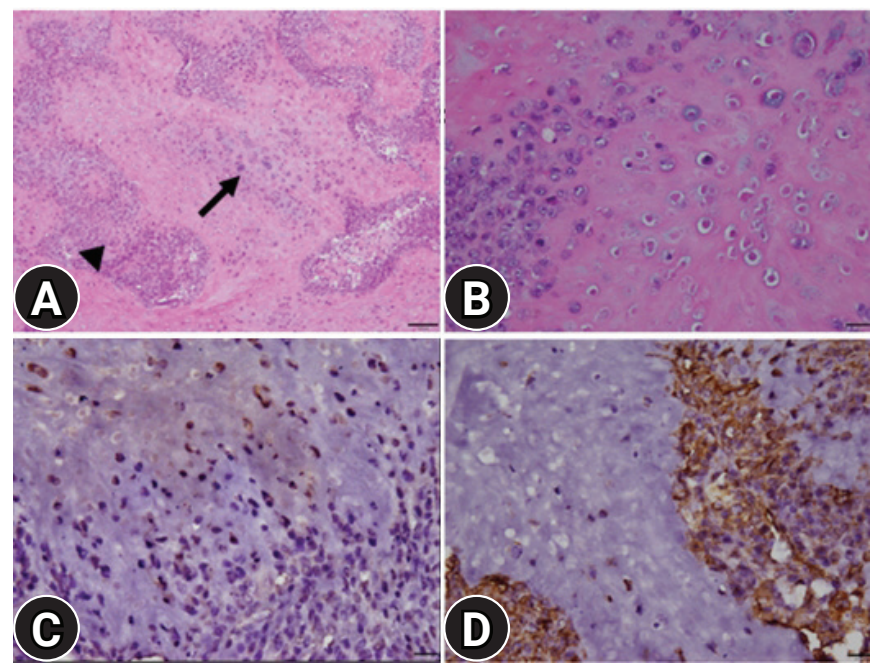

Fig. 2. Histopathological findings. (A) Biphasic patterns of cartilage (arrow) and undifferentiated mesenchymal tissues (arrowhead) (H\&E, scale bar: $100 \mu \mathrm{m})$. (B) Neoplastic chondrocytes are embedded in an eosinophilic or basophilic cartilaginous matrix (H\&E, scale bar: $20 \mu \mathrm{m}$ ). (C) Neoplastic chondrocytes show the immunoreactivity for the S-100 protein, whereas (D) the mesenchymal cells exhibit strong immunoreactivity for vimentin (scale bar: $20 \mu \mathrm{m})$.

cleated giant cells were present within the mass. Severe multifocal necrosis was also present around the mass, particularly in the peripheral area. The cartilaginous materials and lacunae stained greenish-blue, and the nuclei of chondrocytes stained red in contrast to the Alcian blue stain. Through immunohistochemical analysis, most neoplastic cells forming multifocal cartilaginous islands were strongly positive for the S-100 protein (Fig. 2C); the surrounding mesenchymal cells were strongly positive for vimentin (Fig. 2D).

Chondrosarcomas usually occur in older dogs from large breeds, and there is no sex predilection. To the best of the authors' knowledge, this is the first report of mesenchymal chondrosarcoma occurring in the maxillary gingiva of a small-breed Maltese dog.

In dogs, as in humans, chondrosarcomas more often involve the flat bones than the long bones. In a retrospective study of 97 dogs with chondrosarcomas, the nasal cavity was the most common site $(28.8 \%)$, followed by the ribs (17.5\%), appendicular skeleton (17.5\%), extraskeletal sites (13\%), and facial bones (9\%) [3]. In another study, 31 out of 35 cases (88.6\%) involved the flat bones, predominantly the ribs, turbinates, or pelvis [2].

Extraskeletal chondrosarcomas are rare soft tissue sarcomas arising from soft tissues and visceral organs [5]. In dogs, approximately $1 \%$ of chondrosarcomas occur in extraskeletal locations, including the lungs, retroperitoneum, omentum, and 
spleen [6-9].

Histologically, two types of chondrosarcoma are recognized: conventional, the most common type found in skeletal tumors, and mesenchymal, a rarer type found more often in the extraskeletal sites [10]. Myxoid or conventional chondrosarcoma consists of spindle-shaped cells trapped within a basophilic hyaline matrix. Mesenchymal chondrosarcoma has been described as having two distinct histologic patterns. The first is moderate to well-differentiated chondroid tissue, while the second is described as undifferentiated malignant mesenchymal cells arranged in sheets [1]. The histological appearance of the oral mass, in this case, was similar to that described for mesenchymal chondrosarcoma in humans and dogs.

The diagnosis of biopsy specimens remains a challenge because of the variable tumor components, and specimens may contain only one of the two elements. The absence of cartilage in the biopsy sample can confuse this tumor with other small, round, blue-cell tumors. The histological differential diagnosis of mesenchymal chondrosarcoma included lymphoma, neuroblastoma, small cell osteosarcoma, and embryonal rhabdomyosarcoma [11]. Large samples of healthy tissue at the edge and center of the lesion will increase the diagnostic yield. Moreover, typical immunohistochemistry findings include the positivity of the mesenchymal portion for the vimentin and the positivity of the cartilaginous regions for the S- 100 protein. In this case, the requested mass was relatively large and contained two elements essential for diagnosis. Moreover, the mass showed similar immunohistochemical findings to those previously reported [12].

In a diagnostic examination for chondrosarcomas, radiographs only provide the anatomical location of the tumor [13]. Computed tomography can evaluate the degree of bone invasion, and magnetic resonance imaging can reflect the increased aqueous contents of abundant neoplastic cartilage matrix in a T2-weighted hyperintense area, particularly in well-differentiated low-grade chondrosarcoma, as reported in humans [13]. The dog, in this case, was examined using only radiographs, so no evidence of bony involvement was observed within the lesion.

Extraskeletal chondrosarcomas occurring in the oral cavity are exceedingly rare. In a previous study, among 109 malignant oral neoplasms in dogs, none were classified as chondrosarcoma [14]. Extraskeletal chondrosarcoma of the gingiva has been reported in rabbits and humans [15]. In rabbit chondrosarco$\mathrm{ma}$, the tumor invaded the nasal cavity and the survival time after diagnosis was 15 days [15].

In the present case, the dog only had a portion of the mass removed for biopsy; no other aggressive treatment, such as radical surgery with a wide margin or systemic chemotherapy, was per- formed. After diagnosis, the dog was treated only with antibiotics, and the remaining tumor recurred more significantly. The prognosis was poor in this case, and the dog died two months after diagnosis. Although mesenchymal chondrosarcoma of the maxillary gingiva is rare, clinicians and pathologists should be aware of this rare entity when making a differential diagnosis of oral tumors.

\section{ORCID}

So-Jeong Yim, https://orcid.org/0000-0002-5295-0783

Jinyong Lee, https://orcid.org/0000-0003-3612-7268

Jae-Hoon Kim, https://orcid.org/0000-0002-4410-9126

Ji-Youl Jung, https://orcid.org/0000-0002-5938-2029

\section{References}

1. Thompson KG, Dittmer KE. Tumors of Bone. In: Meuten DJ (ed.). Tumors in Domestic Animals. 5th ed. pp. 394-400, Wiley Blackwell, Ames, 2017.

2. Brodey RS, Sauer RM, Medway W. Canine bone neoplasms. J Am Vet Med Assoc 1963;143:471-495.

3. Popovitch CA. Weinstein MJ, Goldschmidt MH, Shofer FS. Chondrosarcoma: a retrospective study of 97 dogs (19871990). J Am Anim Hosp Assoc 1994;30:81-85.

4. Koch BB, Karnell LH, Hoffman HT, Apostolakis LW, Robinson RA, Zhen W, Menck HR. National cancer database report on chondrosarcoma of the head and neck. Head Neck 2000; 22:408-425.

5. Chikata S, Nakamura S, Katayama R, Yanagisawa S, Matsuo Y, Yamane I, Takahashi K. Primary chondrosarcoma in the liver of a dog. Vet Pathol 2006;43:1033-1036.

6. Patnaik AK. Canine extraskeletal osteosarcoma and chondrosarcoma: a clinicopathologic study of 14 cases. Vet Pathol 1990;27:46-55.

7. LaRock RG, Ginn PE, Burrows CF, Newell SM, Henson KL. Primary mesenchymal chondrosarcoma in the pericardium of a dog. J Vet Diagn Invest 1997;9:410-413.

8. Rhind SM, Welsh E. Mesenchymal chondrosarcoma in a young German shepherd dog. J Small Anim Pract 1999;40: 443-445.

9. Miller JM, Walshaw R, Bourque AC. Primary splenic mesenchymal chondrosarcoma in a dog. Can Vet J 2005;46:163-165.

10. Casadei R, Ricci M, Ruggieri P, Biagini R, Benassi S, Picci P, Campanacci M. Chondrosarcoma of the soft tissues: two different sub-groups. J Bone Joint Surg Br 1991;73:162-168.

11. Shakked RJ, Geller DS, Gorlick R, Dorfman HD. Mesenchy- 
mal chondrosarcoma: clinicopathologic study of 20 cases. Arch Pathol Lab Med 2012;136:61-75.

12. Huang WC, Hu KY, Kuo KT, Tsai CC. Mesenchymal chondrosarcoma of the maxilla: case report and clinicopathologic review. Tzu Chi Med J 2013;25:55-57.

13. Chowdhury A, Kalsotra P, Bhagat DR, Sharma P, Katoch P. Chondrosarcoma of the maxilla-recurrent. JK Sci 2008;10:9496.
14. Mikiewicz M, Paździor-Czapula K, Gesek M, Lemishevskyi V, Otrocka-Domagała I. Canine and feline oral cavity tumours and tumour-like lesions: a retrospective study of 486 cases (2015-2017). J Comp Pathol 2019;172:80-87.

15. Miwa Y, Nakata M, Takimoto H, Chambers JK, Uchida K. Spontaneous oral tumours in 18 rabbits (2005-2015). J Small Anim Pract 2021;62:156-160. 